\title{
Cardiac Ischemia Associated With Marijuana Use in an Adolescent
}

Matthew D. Schreier ${ }^{1}$, Collette Williams ${ }^{2}$, T. Marsha Ma ${ }^{3}$

1. Internal Medicine-Pediatrics, Ohio State University/Nationwide Children's Hospital, Columbus, USA 2. Internal Medicine-Pediatrics, Loyola University Medical Center, Maywood, USA 3. Pediatric Cardiology, Loyola University Medical Center, Chicago, USA

Corresponding author: Matthew D. Schreier, matthew.schreier@osumc.edu

\begin{abstract}
With the increasing use of marijuana globally, including in pediatric populations, healthcare providers see more adverse health effects associated with this substance. This report details a case presentation of cardiac ischemia in an adolescent male associated with marijuana use. The patient presented with palpitations and chest pain shortly after consuming marijuana. Clinical workup demonstrated anterior ST-segment elevations and troponin elevation with no inflammatory marker elevation or findings of myocarditis on MRI. Extensive drug screening was only positive for marijuana, with a synthetic THC panel negative. These findings resolved after close observation and the patient was discharged in good clinical condition. This case shows a concerning presentation of marijuana-associated cardiac ischemia in an otherwise healthy adolescent, illustrating a potentially severe health concern with an increasingly common substance and demonstrating the need for pediatric centers to have a high index of suspicion for cardiac causes of chest pain when marijuana ingestion is involved even when there are no prior medical or cardiac risk factors.
\end{abstract}

Categories: Cardiology, Internal Medicine, Pediatrics

Keywords: cardiology, cardiac ischemia, marijuana use, toxicology, adolescent, coronary vasospasm

\section{Introduction}

Marijuana is the most widely-used illicit drug in the United States, with nearly 22.2 million users each month and $38 \%$ of high school students reporting marijuana use [1]. Now increasingly championed for its positive health effects, it also possesses an array of adverse effects. While the neuropsychiatric, gastrointestinal, and pulmonary side effects are more well-known, the cardiovascular effects of marijuana still largely have yet to be elucidated, and those that are reported are largely in the adult population [2-5]. Here we present a case report of an otherwise-healthy 17-year-old male presenting with signs of ischemic cardiac disease shortly after ingestion of marijuana.

Received 06/23/2020 Review began 07/13/2020 Review ended 07/18/2020 Published 08/11/2020

\section{○ Copyright 2020}

Schreier et al. This is an open access article distributed under the terms of the Creative Commons Attribution License CC-BY 4.0., which permits unrestricted use, distribution, and reproduction in any medium, provided the original author and source are credited.

\section{Case Presentation}

A 17-year-old Hispanic male with no prior medical, cardiac, or known substance abuse history and no familial cardiac history presented to an outside hospital approximately 12 hours after feeling a sensation of "heart pounding", chest pain, and dizziness that began a few hours after work as a furniture mover. The chest pain was localized to his left-central chest and radiated with pain and numbness to his jaw and lower face. The patient was hemodynamically stable with no notable vital sign changes. He was noted to have an elevated qualitative iStat troponin and electrocardiogram (ECG) showing ST-segment elevations in V2 and V3, but no other lab abnormalities including a normal erythrocyte sedimentation rate (ESR), C-reactive protein (CRP), and white blood cell (WBC) count. The patient reported that he had used marijuana via a vaporizer three-to-four hours before the incident. He had no recent illnesses and reported no sick contacts, recent travel, or use of any other substances including cocaine. He was placed on telemetry for the duration of his admission.

On admission to our institution, approximately 24 hours after the initiation of symptoms, his troponin I level was elevated to $4.54 \mathrm{ng} / \mathrm{mL}$ (normal range $0.00-0.04 \mathrm{ng} / \mathrm{mL}$ ), his ECG was notable for persistent STsegment elevation in V2 and V3, CRP and ESR were both within normal limits $(0.5 \mathrm{mg} / \mathrm{dL}$ and $4 \mathrm{~mm}$, respectively), and his creatine kinase, creatinine, and serum lipids were within normal limits (Figure 1). His urine drug screen was positive for cannabinoids and negative for cocaine and opiates. Screening for synthetic cannabinoids was also negative. An echocardiogram on admission showed no abnormal findings, and a respiratory panel was done with no viruses detected. He had no murmurs or extra sounds on cardiac exam and his physical exam was otherwise unremarkable. The patient at this time reported continued chest pain, but his palpitations and dizziness had resolved. 


\section{Cureus}

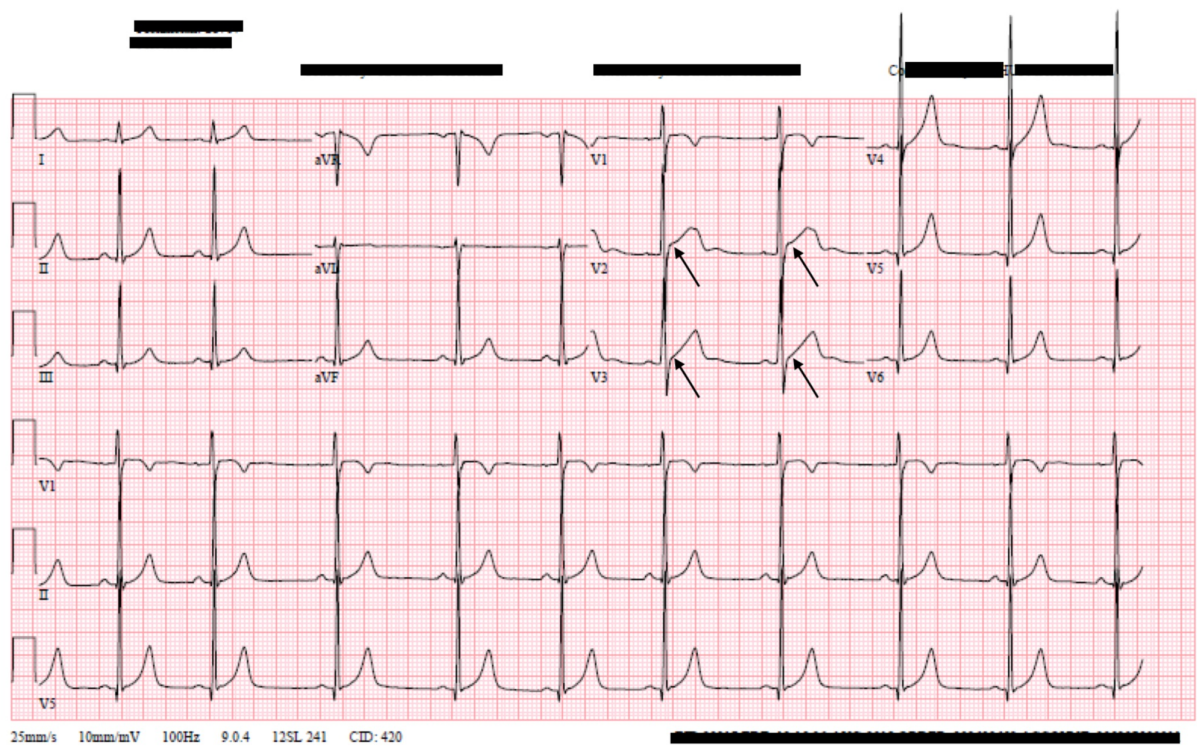

FIGURE 1: Admission ECG demonstrating ST-elevations in V2 and V3 ECG, electrocardiogram

One day into hospitalization (two days after initiation of symptoms), his troponin I had downtrended to 3.09 $\mathrm{ng} / \mathrm{mL}$ and no acute events were noted on telemetry. The patient reported improvement in his clinical condition, with a decrease in chest pain and no recurrences of palpitations. Vital signs remained stable throughout admission. A cardiac MRI was performed to rule-out inflammatory or structural heart disease and showed no evidence of inflammation, fibrosis, myocarditis, infarction, or altered perfusion as well as no anomalous origins of coronary arteries.

By hospital day two, his troponin I had decreased to $2.62 \mathrm{ng} / \mathrm{mL}$, he had no recurrence of chest pain or palpitations, and he was discharged home after a discussion on the importance of avoiding marijuana and with the plan to follow-up as an outpatient (Figure 2).

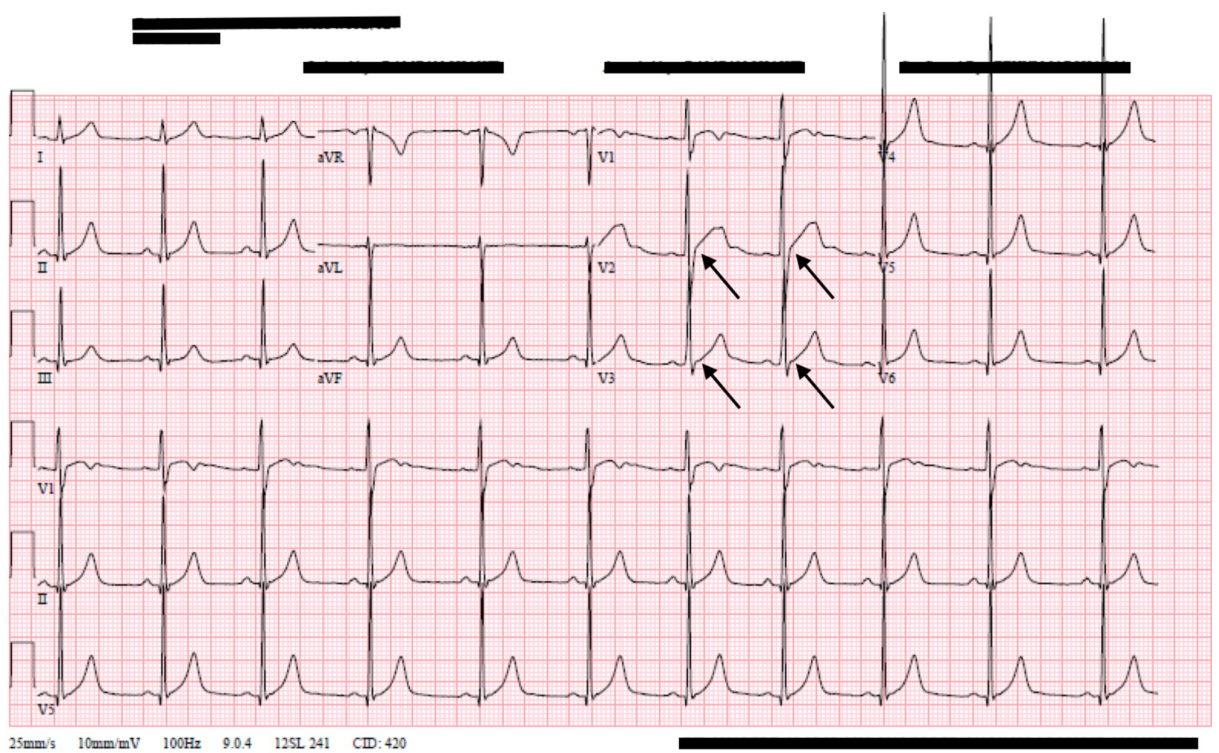

FIGURE 2: Repeat ECG demonstrating improvement in ST-elevations in $\mathrm{V} 2$ and resolution of ST-elevations in V3

ECG, electrocardiogram

At one-month outpatient follow-up, the patient reported no recurrence of symptoms and at this time had a 
normal troponin I level. Additionally, the importance of avoiding marijuana in the future was re-iterated and drug abuse counseling was recommended.

\section{Discussion}

The lack of inflammatory markers in concert with ST-segment elevations and an elevated troponin I level indicate an ischemic etiology of chest pain, and the close proximity of marijuana usage to symptoms suggests a diagnosis of marijuana-induced vasospasm of the coronary arteries.

While the cardiovascular effects of marijuana still have yet to be fully elucidated, it has been shown to have ischemic, arrhythmogenic, and possibly inflammatory effects on the heart. These are largely seen in adults, consistent with the expected higher burden and prevalence of coronary artery disease in the adult population, but these manifestations are being seen in the pediatric and young adult populations as well. Several case reports describe patients meeting criteria for myocardial infarction by ECG and cardiac enzymes after marijuana ingestion in young adulthood with no baseline coronary artery disease on angiography or other substances detected on drug screen [6-8]. Another case series shows similar findings in young adults after the consumption of synthetic marijuana, except with notable thrombus burden on cardiac angiogram $[9]$.

There are also reports of arrhythmogenesis in young patients associated with marijuana consumption including Brugada-like ECG findings in a previously healthy 19-year-old male, and cardiac arrest with ECGcaptured ventricular fibrillation and baseline QTc prolongation noted after defibrillation in a previously healthy 16-year-old female $[10,11]$.

A prior report also notes cardiac inflammation after marijuana use presenting as recurrent myopericarditis in a 29-year-old previously healthy male with pericardial rub, CRP and troponin I elevation, and ECG demonstrated ST-segment elevation and PR-segment depression [12].

Case reports in adolescent and pediatric populations are slightly more limited, but have noted a 17-year-old male diagnosed with coronary vasospasm hours after marijuana ingestion with normal coronary angiography in a presentation similar to the patient detailed above, and sudden cardiac arrest in two adolescent males with ECG-demonstrated ventricular fibrillation after ingestion of synthetic cannabinoids [13,14].

Diagnostic workup in these patients commonly includes cardiac enzymes, inflammatory markers, ECG, echocardiogram, and commonly coronary angiography and cardiac MRI depending on the clinical presentation. Management was based on the presenting manifestation, ranging from supportive care to invasive cardiac procedures as indicated.

There are several hypotheses for the pathogenesis of the cardiovascular effects of marijuana. The active ingredient of marijuana, tetrahydrocannabinol (THC), binds to two major receptors in the body, the CB1 which is found in the myocardium and vascular endothelium and likely attenuates the cardiovascular effects, and the CB2 receptor [15]. THC is known to increase sympathetic effects and decrease parasympathetic effects via norepinephrine signaling, having been shown elevate heart rates by $20 \%-100 \%$, increase cardiac output by $30 \%$, and raise both systolic and diastolic blood pressures $[10,15]$. It has also been demonstrated to elevate carboxyhemoglobin levels, increasing myocardial oxygen demand and decreasing supply, as well as to decrease time to angina in regular users of the drug $[15,16]$. There is also data to support that, similar to the well-known effects of cocaine on the coronary arteries, marijuana use can lead to coronary vasospasm in otherwise normal coronary arteries shortly after exposure, generating a vasospastic angina akin to that seen in the patient described in this report $[15,16]$. Furthermore, there is data to suggest possible effects of THC leading to plaque instability and a procoagulation state in patients with underlying coronary atherosclerosis and thrombosis $[9,15]$.

Overall, there is increasing evidence for a temporal relationship between marijuana and cardiac ischemia, with one study demonstrating a $4.8 \mathrm{x}$ risk of acute myocardial infarction in the first hour after marijuana ingestion, and another study showing a hazard ratio of 2.5 for cardiovascular death in those who used marijuana less than weekly and a hazard ratio of 4.2 who used it more than weekly $[17,18]$. The data in adult patients demonstrate an overall increase in cardiovascular mortality in marijuana users compared to nonusers [16]. It is also reported that the use of synthetic cannabinoids is independently associated with cardiovascular risk, and that synthetics overall have increased cardiovascular effects [3,9].

\section{Conclusions}

With the increasing use of and access to marijuana both medicinally and recreationally in children and adolescents as well as an increasing volume of health concerns associated with smoking and vaping marijuana, it is becoming increasingly essential to be aware of the possible clinical manifestations that could lead users to seek medical care. Current data, still largely in the form of case reports, strongly suggests a cardiac toxicity associated with cannabinoids. While some of the cardiac effects of marijuana have been previously borne out, presentations of acute coronary syndrome, coronary vasospasm, and certain lifethreatening arrhythmias are still seen largely in the adult population. As coronary artery disease is 
significantly less prevalent in pediatrics, it is essential to have a high index of suspicion in pediatric centers to ensure these cases aren't overlooked, and this case adds to the body of literature that these presentations are not limited to the population that will be presenting to adult centers. In a pediatric patient presenting with chest pain, palpitations, and dyspnea, it is important to keep coronary artery disease and new-onset arrhythmias on the differential diagnosis, even if there are no personal or familial cardiac risk factors. Marijuana use should be considered in this differential diagnosis. Even if initial testing for marijuana is negative, consideration can be made to test for newer synthetic cannabinoids if there is a reason for suspicion. Furthermore, when providers are counseling pediatric patients about substance use, it is important to pay attention to these often less-known medical complications, as these may prove to have significant effects on morbidity and mortality.

\section{Additional Information \\ Disclosures}

Human subjects: Consent was obtained by all participants in this study. Conflicts of interest: In compliance with the ICMJE uniform disclosure form, all authors declare the following: Payment/services info: All authors have declared that no financial support was received from any organization for the submitted work. Financial relationships: All authors have declared that they have no financial relationships at present or within the previous three years with any organizations that might have an interest in the submitted work. Other relationships: All authors have declared that there are no other relationships or activities that could appear to have influenced the submitted work.

\section{References}

1. "What You Need to Know About Marijuana Use in Teens, CDC." Centers for Disease Control and Prevention, Centers for Disease Control and Prevention. (2017). Accessed: April 13, 2015: http://www.cdc.gov/marijuana/factsheets/teens.htm.

2. Thomas G, Kloner RA, Rezkalla S: Adverse cardiovascular, cerebrovascular, and peripheral vascular effects of marijuana inhalation: what cardiologists need to know. Am J Cardiol. 2014, 113:187-190. 10.1016/j.amjcard.2013.09.042

3. Hall W, Degenhardt L: Adverse health effects of non-medical cannabis use . Lancet. 2009, 374:1383-1391. 10.1016/S0140-6736(09)61037-0

4. Sorenson CJ, DeSanto K, Borgelt L, Phillips KT, Monte AA: Cannabinoid hyperemesis syndrome: diagnosis, pathophysiology, and treatment - a systematic review. J Med Toxicol. 2017, 13:71-87. 10.1007/s13181-0160595-z

5. Jouanjus E, Leymarie F, Tubery M, Lapeyre-Mestre M: Cannabis-related hospitalizations: unexpected serious events identified through hospital databases. Br J Clin Pharmacol. 2010, 71:758-765. 10.1111/j.13652125.2010.03897.x

6. Safaa AM, Markham R, Jayasinghe R: Marijuana-induced recurrent acute coronary syndrome with normal coronary angiograms. Drug Alcohol Rev. 2012, 31:91-94. 10.1111/j.1465-3362.2011.00327.x

7. Gunawardena MM, Rajapakse S, Herath J, Amarasena N: Myocardial infarction following cannabis induced coronary vasospasm. BMJ Case Rep. 2014, 2014:bcr2014207020. 10.1136/bcr-2014-207020

8. Casier I, Vanduynhoven P, Haine S, Vrints C, Jorens PG: Is recent cannabis use associated with acute coronary syndromes? An illustrative case series. Acta Cardiol. 2014, 69:131-136. 10.2143/AC.69.2.3017293

9. Ul Haq E, Shafiq A, Khan AA, Awan AA, Ezad S, Minteer WJ, Omar B: Spice (synthetic marijuana) induced acute myocardial infarction: a case series. Case Rep Cardiol. 2017, 2017:9252463. 10.1155/2017/9252463

10. Pratap B, Korniyenko A: Toxic effects of marijuana on the cardiovascular system . Cardiovasc Toxicol. 2012, 12:143-148. 10.1007/s12012-011-9150-y

11. Davis C, Boddington D: Teenage cardiac arrest following abuse of synthetic cannabis. Heart Lung Circ. 2015, 24:e162-e163. 10.1016/j.hlc.2015.04.176

12. Rodríguez-Castro CE, Alkhateeb H, Elfar A, Saifuddin F, Abbas A, Siddiqui T: Recurrent myopericarditis as a complication of marijuana use. Am J Case Rep. 2014, 15:60-62. 10.12659/AJCR.889808

13. Basnet S, Mander G, Nicolas R: Coronary vasospasm in an adolescent resulting from marijuana use . Pediatr Cardiol. 2009, 30:543-545. 10.1007/s00246-009-9384-7

14. Alam S, Anderson S, Deshpande S: Sudden cardiac arrest in the setting of synthetic marijuana use: two interesting cases posing a difficult but important diagnostic dilemma. Pediatrics. 2018, 142:1-2. 10.1542/peds.142.1_MeetingAbstract.478

15. Franz CA, Frishman WH: Marijuana use and cardiovascular disease . Cardiol Rev. 2016, 24:158-162. 10.1097/CRD.0000000000000103

16. Rezkalla S, Stankowski R, Kloner RA: Cardiovascular Effects of Marijuana . J Cardiovasc Pharmacol Ther. 2016, 21:452-455. 10.1177/1074248415627874

17. Mittleman MA, Lewis RA, Maclure M, Sherwood JB, Muller JE: Triggering myocardial infarction by marijuana. Circulation. 2001, 103:2805-2809. 10.1161/01.cir.103.23.2805

18. Mukamal KJ, Maclure M, Muller JE, Mittleman MA: An exploratory prospective study of marijuana use and mortality following acute myocardial infarction. Am Heart J. 2008, 155:465-470. 10.1016/j.ahj.2007.10.049 